\title{
Minimal dissipation model for bipartite quantum systems at finite temperature
}

\author{
Alexander Ramírez Bolaños, ${ }^{1}$ Héctor J. G. Moreno Carrión, ${ }^{2,3}$ and Thomas Gorin ${ }^{1}$ \\ ${ }^{1}$ Departamento de Fúsica, Universidad de Guadalajara, \\ Blvd. Marcelino García Barragan y Calzada Olímpica, C.P. 44840, Guadalajara, Jalisco, México \\ ${ }^{2}$ Centro de Investigación en Ciencias, Universidad Autónoma del Estado de Morelos, Cuernavaca, Morelos, México \\ ${ }^{3}$ Instituto de Ciencias Físicas, Universidad Nacional Autónoma de México, Cuernavaca, Morelos, México
}

\begin{abstract}
We consider the reduced dynamics in a bipartite quantum system (consisting of a central system and an intermediate environment) coupled to a heat bath at finite temperature. To describe this situation, in the simplest possible - yet physically meaningful way, we introduce the "depolarizing heat bath" as a new minimal dissipation model. We conjecture that at sufficiently strong dissipation, any other dissipation model implemented in the form of a Markovian quantum master equation will yield the same reduced dynamics of the central system, as the minimal model.

To support this conjecture, we study a two-level system coupled to an oscillator mode. For the coupling between the two parts, we consider the Jaynes-Cummings or a dephasing coupling, while the coupling to the heat bath is modeled by the quantum optical or the Caldeira-Leggett master equation (neglecting any direct coupling between central system and heat bath). We then provide ample numerical evidence, for both, model-independence and accuracy of the depolarizing heat bath model. Alongside with our study, we investigate different regimes, where the strong coupling condition leads to coherence and/or population stabilization.
\end{abstract}

\section{INTRODUCTION}

The concept of a generic, impartial heat bath plays an important role in equilibrium statistical mechanics, where it is used to keep the temperature of thermodynamic systems (classical or quantum) well defined and constant, without changing the (thermodynamic) properties of these systems themselves. In the case of the canonical ensemble, for instance, one assumes the system to be in contact with a much larger second system (the environment), such that both systems together form an isolated composite system, treatable as a microcanonical ensemble. Then, in order to act as a proper heat bath, the coupling between system and environment and the environment itself should be such that (i) the dynamical properties of the system remain unchanged and (ii) thermal relaxation still occurs [1]. As a consequence, only two quantities, temperature and coupling strength, are sufficient to completely specify the effect of that heat bath on the system.

The description of open quantum systems and their relaxation processes is usually more complicated. Even in the simplest case, where a description in terms of a Markovian quantum master equation is possible [2, 3], the required dissipation (Lindblad) terms, will depend on the dynamics of the system, the coupling operator(s), and the way these interact with the degrees of freedom of the heat bath [4]. Moreover, at the end of the relaxation process, the system returns to equilibrium states, which are thermal mixtures of the eigenstates of some modified system Hamiltonian [4]. This implies changes in the thermodynamic properties of the system, in contrast to the setting presented above.

In order to resolve this conflicting situation, we introduce an intermediate system, which interacts with the heat bath as postulated by an ideal thermal contact, while allowing any type of interaction with the central system. Formally, this can be done by selecting appropriately some of the degrees of freedom of the environment, treating them as the intermediate system; see for instance the Refs. [5-8]. We then use the "depolarizing heat bath" model for describing the effect of the external heat bath on the intermediate system, which comes as close as possible to the idea of an impartial thermal contact. Its high temperature limit has been derived in the context of random matrix models for decoherence [9]. In the context of quantum thermal machines [8, 10, 11], this model is known as "reset model"; as such it has been introduced in [10] for describing two-level systems coupled to thermal reservoirs.

We then consider dissipative bipartite systems, consisting of a two-level system (qubit) and a harmonic oscillator mode, which plays the role of the intermediate system in the setup mentioned above. We show that for different types of couplings (between qubit and oscillator mode) and different dissipation models, the reduced dynamics of the qubit always tends to the of the depolarizing heat bath, as the coupling to the heat bath (measured by the dissipation rate) is increased.

As the bipartite system we consider a central two-level system (qubit), coupled to a harmonic oscillator mode as "intermediate system". Qubit and oscillator are coupled either by a Jaynes-Cummings (JC), or a dephasing (D) coupling. As heat baths or dissipation models, we consider the quantum optical (QO) and the Caldeira-Leggett (CL) master equation. The JC coupling together with the QO master equation, represents one of the paradigmatic models in quantum optics, the dissipative JaynesCummings model [12-15]; see also the recent special issue, Ref. [16]. The dephasing coupling case has experimental relevance, for instance as a simplified description of the dynamics of defect centers taking into account the coupling to lattice phonons [17]. The CL master equation finally is another paradigmatic model for quantum 
dissipation, as it provides the quantum analog to the classical damped harmonic oscillator in the underdamped regime [18, 19], applicable for instance to quantum Brownian motion.

The depolarizing heat bath master equation (or more precisely, its high temperature limit) has been derived in the context of random matrix models for decoherence [9], considering the reduced dynamics of one system described by a random matrix ensemble, in the presence of an environment, described by a similar but statistically independent ensemble. It has then been used, to describe a dissipative bipartite system with the near environment modeled by random matrix theory [20, 21]. There, it was shown that the decoherence rate in the central system may be inversely proportional to the dissipation rate in the near environment. Related phenomena of coherence and/or population stabilization have been observed in other dissipative bipartite systems including the dissipative JC model: The first reference is probably Ref. [22], but more explicit accounts of the matter can be found in Ref. [23] and very recently in Ref. [24]. Finally, the case of a deterministic quantum chaotic spin system has been studied in [25].

The paper is organized as follows: In the next section, Sec. II, the different models are introduced. They all have the same structure, a quantum master equation for the mixed quantum state of central system and near environment. In Sec. III, we present numerical simulations for the different models, focussing on the accuracy of our "depolarizing heat bath" model, and the above mentioned stabilization effect. Finally, we present our conclusions in Sec. IV.

\section{GENERAL MODEL}

In this paper, we consider bipartite open quantum systems, strongly coupled to an external heat bath. Typical examples are (i) the dissipative Jaynes-Cummings (JC) model, and (ii) the Caldeira-Leggett (CL) model coupled to an internal degree of freedom. We distinguish between the central system, assumed to be a two-level system (qubit) and the much larger near environment, here chosen as a harmonic oscillator mode with angular frequency $w_{\mathrm{e}}$. We assume that only the near environment is coupled to the heat bath. The evolution of the bipartite system is modeled via a quantum master equation, which consists of a Hamiltonian part describing the dynamics of central system and near environment, and a dissipative part, which describes the effect of the heat bath on the near environment. The dissipative part, has two main control parameters, the temperature and the coupling strength.

Dividing the quantum master equation in original physical units by $\hbar w_{\mathrm{e}}$ and switching from physical time $t_{\mathrm{ph}}$ to the dimensionless time $t=w_{\mathrm{e}} t_{\mathrm{ph}}$, we are left with a differential equation of the following form

$$
\dot{\varrho}=-i\left[H_{x}, \varrho\right]-\frac{\kappa}{2} \mathcal{D}[\varrho],
$$

where $H_{x}$ denotes the Hamiltonian which would govern the dynamics of central system and near environment, if they were perfectly isolated, and $\mathcal{D}[\varrho]$ denotes the dissipation term, which would model the effect of the heat bath on the near environment, in the absence of any central system. Here, we introduced the parameter $\kappa$ as a dimensionless dissipation rate, i.e. $\kappa=\gamma / w_{\mathrm{e}}$, where $\gamma$ is the physical energy dissipation rate.

For the Hamiltonian $H_{x}$, the following two models will be considered: (i) The Jaynes-Cummings model $(x=$ $\mathrm{JC}$,

$$
H_{J C}=\frac{\Delta}{2} \sigma_{z}+\hat{a}^{\dagger} \hat{a}+g\left(\sigma_{+} \otimes \hat{a}+\sigma_{-} \otimes \hat{a}^{\dagger}\right),
$$

where we eliminated the zero-point energy term, since it has no effect for the dynamics of the density matrix $\varrho$. With the energy difference in the two level system being denoted by $\hbar w_{\mathrm{a}}$, the dimensionless parameter $\Delta$ becomes $\Delta=w_{\mathrm{a}} / w_{\mathrm{e}}$. Similarly, the coupling parameter $g$ is defined as $g=\Omega /\left(2 w_{\mathrm{e}}\right)$, where $\Omega$ is the Rabi-frequency of the original JC model. Note that in order to arrive at the JC model, a rotating wave approximation must be applied, which is justified if the level spacing in the qubit is equal or close to the boson energy of the harmonic oscillator. In our case, this means for the relative detuning $\delta=\Delta-1$, it must hold that $|\delta| \ll 1$.

(ii) The harmonic oscillator with dephasing coupling $(x=\mathrm{D})$ is defined analogously, using the same units for time and energy as above.

$$
H_{\mathrm{D}}=\frac{\Delta}{2} \sigma_{z}+\hat{a}^{\dagger} \hat{a}+\frac{g}{\sqrt{2}} \sigma_{z} \otimes\left(\hat{a}+\hat{a}^{\dagger}\right) .
$$

\section{A. Quantum optical and Caldeira-Leggett master equations}

In this section, we introduce the quantum optical (QO) master equation alongside with the Caldeira-Leggett (CL) master equation. For convenience, we use the term "quantum optical" for the master equation of the dissipative Jaynes-Cummings model, even though there exist of course many different types of quantum optical master equations. The $\mathrm{QO}$ master equation is meant to describe the coupling of a single cavity mode to an ensemble of electromagnetic (photon) modes in thermal equilibrium. The coupling is assumed to occur due to imperfect mirrors in the cavity, often quantified by a finite quality factor or equivalently a finite dissipation rate. For the QO master equation, the dissipation term $\mathcal{D}[\varrho]$ in Eq. (1) is replaced by

$$
\begin{aligned}
\mathcal{D}_{\mathrm{QO}}[\varrho] & =(\bar{n}+1) \mathcal{D}_{0}[\varrho]+\bar{n}\left(\hat{a} \hat{a}^{\dagger} \varrho-2 \hat{a}^{\dagger} \varrho \hat{a}+\varrho \hat{a} \hat{a}^{\dagger}\right), \\
\mathcal{D}_{0}[\varrho] & =\hat{a}^{\dagger} \hat{a} \varrho-2 \hat{a} \varrho \hat{a}^{\dagger}+\varrho \hat{a}^{\dagger} \hat{a} .
\end{aligned}
$$


Here, $\mathcal{D}_{\mathrm{QO}}$ is the finite temperature and $\mathcal{D}_{0}$ the zerotemperature heat bath (bare vacuum), and $\bar{n}$ is the average number of oscillator modes occupied at the given temperature.

The CL master equation [18] is meant to describe quantum Brownian motion, i.e. a heavy but still quantum mechanical particle in an harmonic potential, subject to dissipation due to frequent collisions with the particles of a finite temperature background gas. In this case, the model has a precise classical analog, which is the damped harmonic oscillator, with damping (or dissipation) rate $\gamma$. As in the quantum optical case, $\kappa=\gamma / w_{\mathrm{e}}$.

In the case of the CL model, the dissipation term is usually written in terms of physical position and momentum operators, while the temperature enters the expression via a diffusion constant. However, for the sake of a consistent description, we rewrite the CL dissipation term, using the same adimensional quantities as in the QO case. The details can be found in App. B with the result given in Eq. (B2) as reproduced here.

$$
\mathcal{D}_{\mathrm{CL}}[\varrho]=2 i[\hat{x},\{\hat{p}, \varrho\}]+2(2 \bar{n}+1)[\hat{x},[\hat{x}, \varrho]],
$$

where the dimensionless position and momentum operators $\hat{x}$ and $\hat{p}$ are defined such that $\hat{a}=(\hat{x}+i \hat{p}) / \sqrt{2}$. As usual, we denote the commutator (anti-commutator) between two operators $A, B$ as $[A, B]=A B-B A$ $(\{A, B\}=A B+B A)$.

The CL dissipation model is derived in the limit of high temperature, and the resulting master equation is not of Lindblad form. It may thus violate the positivity of the evolving density matrix [26, 27]. However, as long as the temperature is not very small this problem has no significant effect on physical quantities [28].

In both models, $\kappa$ is the dissipation rate in units of the angular frequency $w_{\mathrm{e}}$ of the oscillator mode. It describes the rate of energy loss, when the initial state has higher thermal energy than the respective heat bath; see Fig. 1.

\section{B. Depolarizing heat bath}

We are interested in relaxation processes, where only the central system is taken out of equilibrium. Thus, we assume that the initial state of our bipartite system is a product state of an arbitrary initial state $\varrho_{\mathrm{a}}(0)$ of the central system and a thermal equilibrium state $w_{T}$ of the near environments, who's temperature $T$ agrees with that of the heat bath (see App. A). Without coupling to the central system, that state would then be a equilibrium solution of the master equation. We then concentrate on the reduced dynamics of the central system in the regime of strong coupling between intermediate system and heat bath. In such a situation, we may assume that the details of the dynamics in the intermediate system are not so important than the fact that the intermediate system has a strong tendency to quickly return to the equilibrium state.
To describe this situation, we consider the simplest possible quantum operation, which maps any mixed state directly to the equilibrium state (we call this operation "depolarizing channel"). This operation is turned into a dissipation term in the master equation by using Milburn's theory [29]. Physically, this means that the depolarizing channel is applied to the system with a certain rate, $\gamma_{\mathrm{P}}$, which plays the role of the coupling parameter between near environment and heat bath. Comparing the resulting master equation with that in Eq. (1), we find again that $\kappa=\gamma_{\mathrm{P}} / w_{\mathrm{e}}$ and finally the following dissipation term:

$$
\mathcal{D}_{\mathrm{DH}}[\varrho]=2\left(\varrho-\operatorname{tr}_{\mathrm{e}} \varrho \otimes w_{\mathrm{T}}\right),
$$

where $w_{\mathrm{T}}$ is the finite temperature equilibrium state of the near environment alone. Unfortunately, $\gamma_{\mathrm{P}}$ cannot be directly compared to the energy dissipation rate $\gamma$, as defined in the previous models. It is therefore not clear, how to choose $\gamma_{\mathrm{P}}$ as compared to $\gamma$ such that the previous models really converge to this DH model, at strong coupling. According to Fig. 1, where we study directly the energy dissipation, it seems that $\gamma_{\mathrm{P}}$ and $\gamma$ should simply be equal. However, in subsequent studies concentrating on the reduced dynamics of the central system, it appears that $\gamma_{\mathrm{P}}$ should rather be equal to $\gamma / 2$.

In order to appreciate the impartiality of the $\mathrm{DH}$ model, consider the dynamics of the oscillator mode without coupling to the qubit. In that case, the solution to the master equation (1) with dissipation term $\mathcal{D}_{\mathrm{DH}}$ is of the form $\varrho_{\mathrm{a}}(t) \otimes \varrho_{\mathrm{e}}(t)$, with

$$
\frac{\mathrm{d}}{\mathrm{d} t} \tilde{\varrho}_{\mathrm{e}}(t)=-\kappa\left(\tilde{\varrho}_{\mathrm{e}}(t)-w_{T}\right)
$$

where $\tilde{\varrho}_{\mathrm{e}}(t)$ describes the cavity state in the interaction picture. According to this equation, all matrix elements of $\tilde{\varrho}_{\mathrm{e}}(t)$ converge exponentially towards the matrix elements of the thermal equilibrium state $w_{T}$, with the same rate $\kappa$.

\section{Evolution}

We are mainly interested in the evolution of the qubit (the central system), under the coupling to the cavity mode and the external heat bath as a composite environment. We compute the evolution by numerically solving the master equation (1) with the help of a standard solver for ordinary differential equations. Typically, we use about 20 up to 40 basis states in the Hilbert space of the oscillator. Choosing an initial state of the form $\varrho(0)=\varrho_{\mathrm{a}}(0) \otimes w_{T}$, we obtain the evolution of the full bipartite system as $\varrho(t)$. From this quantity, we compute the state of the two-level system by taking a partial trace over the oscillator mode. 


\section{NUMERICAL RESULTS}

In what follows, we consider the evolution of the qubit under different couplings (Jaynes-Cummings, dephasing) and different dissipation models (quantum-optical, Caldeira-Leggett, and depolarizing heat bath) as introduced in Eqs. (4) and (7). We concentrate on the strong coupling regime, where we expect the depolarizing heat bath (DH) model to be a good substitute for any other model. Thus, the main objective in this section consists in finding numerical evidence that the state evolution in all cases converge to the evolution under the DH model, as $\kappa / g \rightarrow \infty$. Note that we expect this to be valid only if the initial state of the near environment is equal (or at least sufficiently close) to the thermal equilibrium state to be reached at the end of the relaxation process. We solve the master equation numerically as a system of linear differential equations using the eigenstates of the harmonic oscillator mode as an orthonormal basis to expand all operators involved. Unless states otherwise, the basis is limitted to quantum numbers $n \leq n_{\max }=40$.

The second objective addresses the stabilization effect found in some related models: (i) In a random matrix model for dephasing coupling [20, 21], we found the stabilization effect only for $g \ll 1$ and $\kappa / g \gg 1$. On this basis, we could for instance exclude the quantum Zeno effect as a possible explanation for the effect. (ii) For the dissipative JC model [22-24] (JC coupling between qubit and oscillator mode, and quantum optical master equation), the only requirement is $\kappa / g \gg 1$. To these cases, we will add a third: (iii) The harmonic oscillator mode with dephasing coupling to a qubit and quantum optical dissipation, a model which is quite similar to the one considered in Ref. [17]. As we will see below, in this case the only requirement is $\kappa \gg 1$.

\section{A. Dissipation rate}

We would like to make sure that the dissipation rate, controlled in the master equation (1) by the parameter $\kappa$, is the same for all dissipation models, considered. For that reason, we analyze the equilibration of the oscillator mode without coupling to the qubit. We choose the initial state to be a thermal equilibrium state with $\bar{n}(0)=3$ and compute its evolution and average energy $\bar{n}(t)=\left\langle\hat{a}^{\dagger} \hat{a}\right\rangle_{\varrho(t)}$ when the temperature of the heat bath is such that $\bar{n}_{\mathrm{eq}}=1$.

The simplest theoretical expectation for $\bar{n}(t)$ is an exponential decay towards the new equilibrium energy, i.e.

$$
n_{\text {theo }}(t)=\bar{n}_{\mathrm{eq}}+\left[\bar{n}(0)-\bar{n}_{\mathrm{eq}}\right] \mathrm{e}^{-\kappa t} .
$$

This expectation is verified in Fig. 1. In the main panel, we show the behavior of $\bar{n}(t)$ for the quantum optical dissipation model (red solid line); in the inset we show the difference between all three dissipation models and the theoretical expectation, Eq. (9). In that graph, the quantum optical (QO) and the depolarizing heat bath (DH)

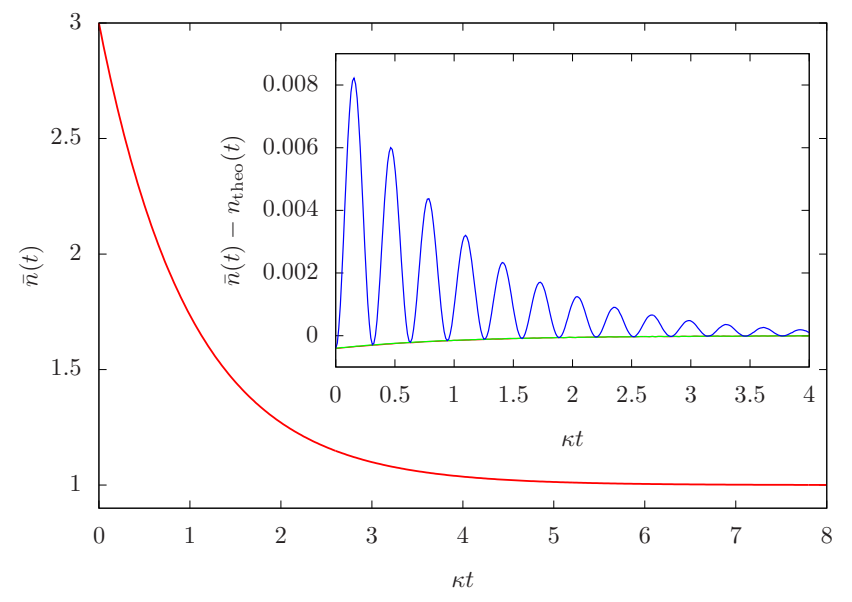

FIG. 1. (Color online) Equilibration without qubit $(g=0)$ for $\kappa=0.1$ and a initial thermal state with $\bar{n}(0)=3$, for the quantum optical (QO) dissipation model. The inset shows the difference between the different dissipation models and the theorical model in Eq. (9): Red line $\left(\mathcal{D}_{\mathrm{QO}}[\varrho]\right.$, quantum optical); blue line $\left(\mathcal{D}_{\mathrm{CL}}[\varrho]\right.$, Caldeira-Leggett); green line $\left(\mathcal{D}_{\mathrm{DH}}[\varrho]\right.$, depolarizing heat bath).

cases are lying almost on top of each other, so that only the green curve can be seen. The Caldeira-Leggett model (blue line) shows clear deviations of the order of one percent. The QO and $\mathrm{DH}$ cases show a small deviation, noticeable at $\kappa$ t close to zero, only. We attribute this to the finite number of basis states $\left(0 \leq n \leq n_{\max }=40\right)$ we have been using for the simulations. The differences in the CL result in the form of damped oscillations, probably are an artefact, related to the Caldeira-Leggett master equation being valid at large temperatures, only.

\section{B. Jaynes-Cummings coupling at zero temperature}

For zero and non-zero temperature, the only relevant energy (frequency) scale is given by $g$. This is due to the fact that the Jaynes-Cummings Hamiltonian in Eq. (2) can be decomposed into two commuting parts, one of which is an observable for the number of excitations in the system: $H_{\text {ext }}=\sigma_{z} / 2+\hat{a}^{\dagger} \hat{a}$. Therefore, if timedependent quantities are plotted against $g t$, the only independent parameters left are the detuning, $(\Delta-1) / g$, and the relative coupling strength to the external heat bath, $\kappa / g$. This is true for all dissipation models discussed in this paper, as given in the Eqs. (4) - (7).

Let us first consider the behavior of the excited state population; this is done in Fig. 2. There, we choose $\varrho_{\mathrm{a}}(0)=|0\rangle\langle 0|$ and select two different values for the detuning, $(\Delta-1) / g=0.8$ (upper panel) and 0.1 (lower panel), where the first case is identical to a case treated in Ref. [24] (Fig. 2 in that reference). We compare the behavior of the excited state population $\left\langle 0\left|\varrho_{\mathrm{a}}\right| 0\right\rangle$, under the quantum optical (solid lines) and the depolarizing 

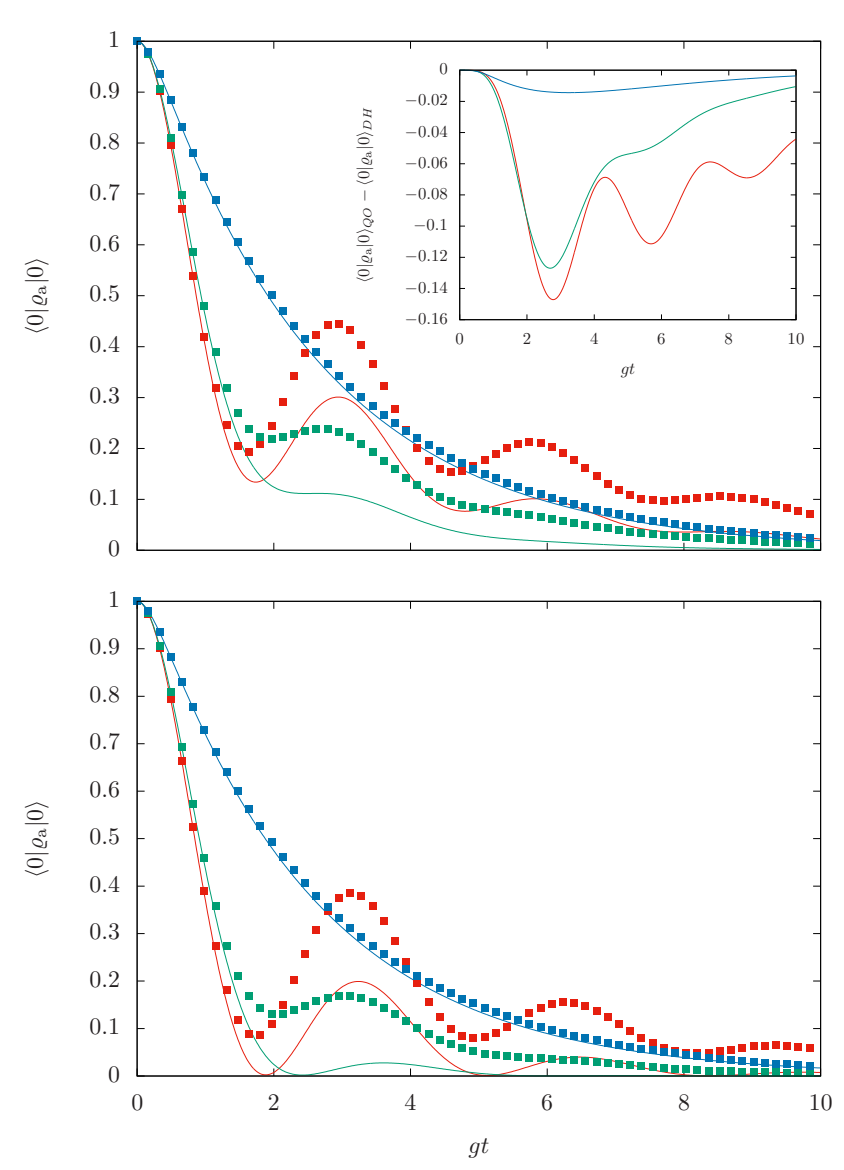

FIG. 2. (Color online) Exited state population as a function of scaled time $g t$ for the dissipative JC model at zero temperature. Comparison between the original quantum optical dissipation (solid lines) and the depolarizing heat bath model (square points). The color coding identifies the different dissipation rates: $\kappa / g=1.0$ (red), 2.0 (green), 10.0 (blue). For the DH model, all rates have been reduced by one half. The upper panel shows the large detuning case, $(\Delta-1) / g=0.8$; in the lower panel $(\Delta-1) / g=0.1$.

heat bath (square points) dissipation model. For $\kappa$ of the order of $g,\left\langle 0\left|\varrho_{\mathrm{a}}\right| 0\right\rangle$ shows rather strong oscillations, which are reproduced qualitatively by the $\mathrm{DH}$ model, but quantitative differences remain. Since the temperature was chosen to be zero, the population always tends to zero at large times.

As expected, we find that both models lead to the same behavior if the coupling to the heat bath is sufficiently strong. Unexpectedly however, in order to achieve that, we had to reduce the dissipation rate for the DH model by a factor of two as compared to the QO model. For the large detuning case, we show the difference between the two dissipation models in the inset of the upper panel, providing clear evidence of the behavior just described. Finally, we find the expected stabilization effect, without much differences between large and small detuning.

Fig. 3 shows the behavior of the coherence (absolute value of the non-diagonal element of the qubit state),
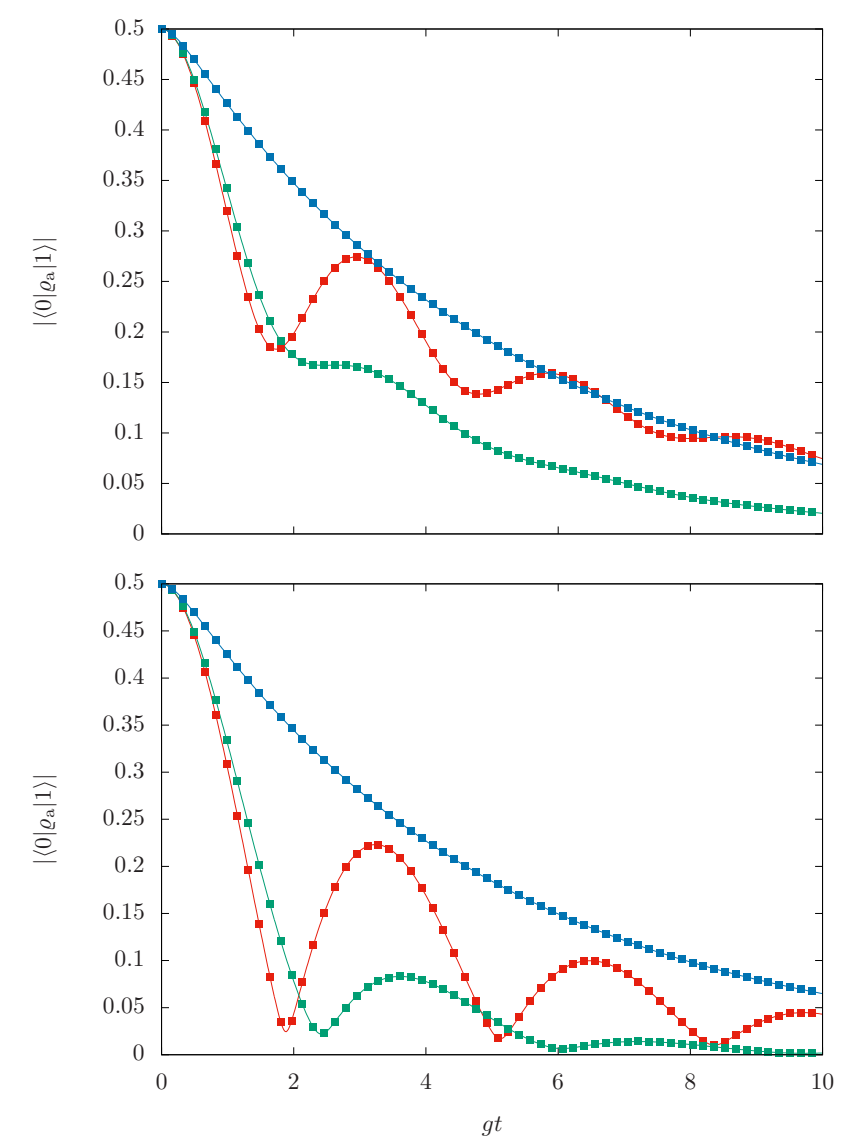

FIG. 3. (Color online) The coherence for an eigenstate of $\sigma_{x}$ as initial state under exactly the same conditions as in Fig. 2. In this case, the QO model (solid lines) and the DH model (square points) yield exactly the same result.

when the initial state is an eigenstate of $\sigma_{x}$,

$$
\varrho_{\mathrm{a}}(0)=\frac{1}{2}\left(\begin{array}{ll}
1 & 1 \\
1 & 1
\end{array}\right)
$$

In this case, the dynamics is exactly the same for both dissipation models (this is no longer true, when the temperature is different from zero). Again, we find a stabilization (slower decay of the coherence), when the coupling to the heat bath is increased.

To summarize, for zero temperature and JaynesCummings coupling, the QO-dynamics converges to the DH-dynamics for the populations (diagonal elements of the reduced qubit state), for the coherence (nondiagonal elements) both models yield exactly the same behavior. We also find the expected stabilization effect at $\kappa>g$ for both, for the diagonal as well as for the non-diagonal elements. In all cases, $\kappa$ had to be divided by two in the $\mathrm{DH}$ model, in order to reach agreement at strong coupling. 


\section{Jaynes-Cummings coupling at finite temperature}

In this section, we compare all three dissipation models, the quantum optical (QO), the depolarizing heat bath (DH) and the Caldeira-Leggett model (CL). For the CL model, the angular frequency $w_{\mathrm{e}}$ of the oscillator mode provides an additional energy scale. Since the model is valid in the underdamped case only (in terms of the classical damped harmonic oscillator), we should not consider the CL model at dissipation rates beyond $\kappa=1$.

Thermal equilibrium in the central system Besides the comparison of the different dissipation models, and the question about the stabilization effect, we may ask whether the coupling between qubit and cavity mode may be considered as a thermal contact. In this case, we would expect that the final state of the bipartite system is a product state with the cavity mode and the qubit in thermal equilibrium states corresponding to the same temperature $T$, established by the external heat bath.

Since we quantify the temperature in terms of the average number of excited modes $\bar{n}=\left\langle\hat{a}^{\dagger} \hat{a}\right\rangle$ at thermal equilibrium (see App. A). Let us assume that the twolevel atom (qubit) reaches an equilibrium state, close to the thermal equilibrium state. According to Eq. (A2), its adimensional inverse temperature is given by

$$
\begin{aligned}
b & =\frac{\hbar w_{\mathrm{e}}}{k_{\mathrm{B}} T}=2 \operatorname{atanh}\left[(2 \bar{n}+1)^{-1}\right] \\
& \Rightarrow \quad\left\langle 0\left|\varrho_{a}^{\mathrm{eq}}\right| 0\right\rangle=\frac{1-\tanh (\Delta b / 2)}{2} .
\end{aligned}
$$

In Fig. 4, we analyze the same cases as in Fig. 2 but at finite temperature, $\bar{n}=1$. This allows us to include results for the Caldeira-Leggett (CL) dissipation model, also. Note however, that we performed all calculations with $g=0.1$, such that for the CL model the permissible values for $\kappa / g$ are limited to $\kappa / g \leq 10$. To our surprise, the CL results are practically indistinguishable from the QO results in this case, except for the strongest dissipation, $\kappa / g=10$, where we find a small difference (see inset of the lower panel).

For clarity, Fig. 4 shows results for the small detuning case $(\Delta-1) / g=0.1$, only. This allows us to plot the curves for $\kappa / g=1.0$ in the upper panel and all others in the lower panel. The results for large detuning, $(\Delta-$ $1) / g=0.8$ (not shown), are very similar.

Qualitatively, we find a very similar behavior as in the zero temperature case: A decay to the equilibrium value, and superimposed oscillations, as long as the dissipation rate $\kappa$ is not too large. Again we observe a stabilization effect, when the dissipation rate is increased. And again, the QO and CL results tend to converge to the DH results (at half the dissipation rate) in the limit of strong dissipation.

In Fig. 4, the equilibrium state is no longer the ground state $|1\rangle$, but a thermal mixture between excited and ground state. The solid black horizontal line, shows the
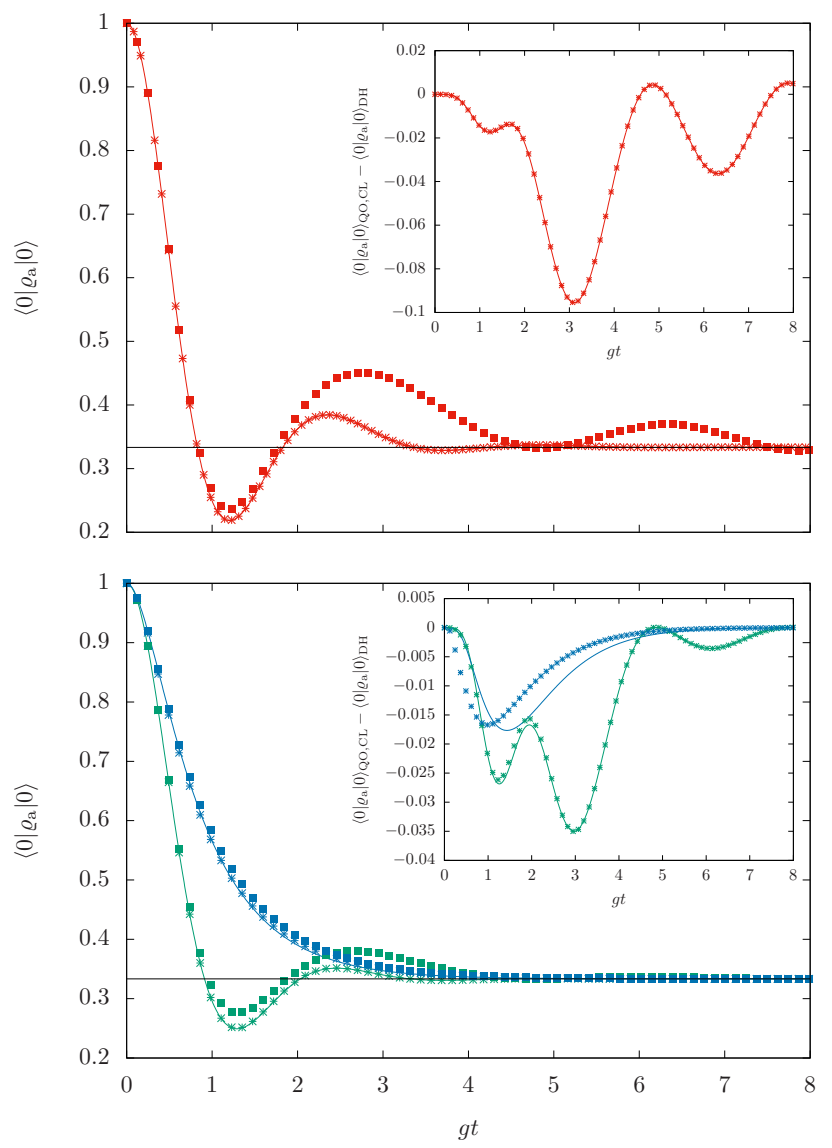

FIG. 4. (Color online) Exited state population as a function of scaled time $g t$ for the dissipative JC model for small detuning $(\Delta-1) / g=0.1$ and finite temperature, $\bar{n}=1.0$. The horizontal black line shows the thermal equilibrium value expected on the basis of Eq. (11). Solid lines show results for the QO model, asterisks those for the Caldeira-Leggett model, and square points those for the DH model. The color coding identifies the different dissipation rates: $\kappa / g=1.0$ (red; upper panel), 2.0 (green; lower panel) and 10.0 (blue; lower panel). Again, for the DH model, all rates have been reduced by one half. In the two insets, we plot the difference between the results for both models.

probability of the qubit to be in the excited state, provided it is in a thermal state, in thermal equilibrium with the cavity mode, according to Eq. (11). The figure clearly provides evidence that the $\mathrm{JC}$ coupling between qubit and oscillator mode, acts indeed as a thermal contact, which forces the qubit into a thermal equilibrium state at the same temperature than external heat bath and oscillator mode.

To conclude this section about the Jaynes-Cummings model at finite temperature, we consider again the behavior of the coherence. In the two remaining figures to be shown, we remove the CL dissipation case, since in the region where it is valid $(\kappa \leq 1)$, the results are practically indistinguishable from the QO case.

Fig. 5 shows the coherence, for an eigenstate of $\sigma_{x}$ as 

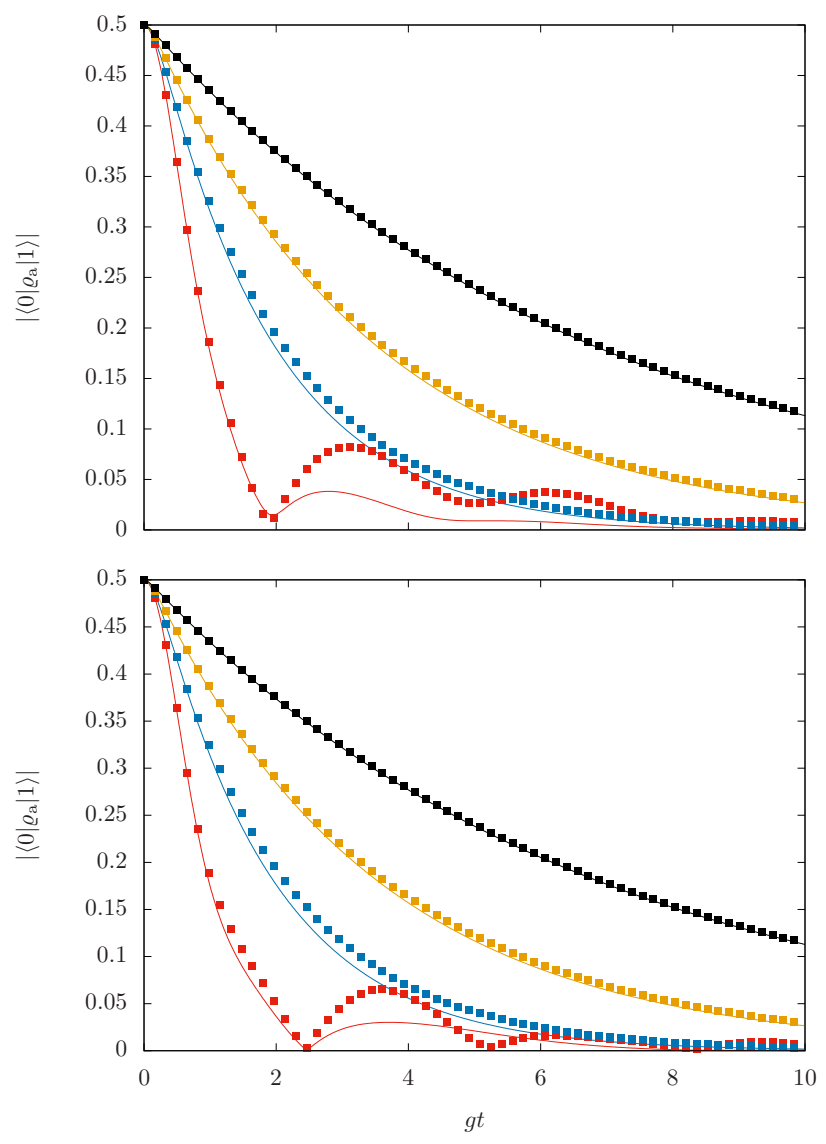

FIG. 5. (Color online) Coherence for an eigenstate of $\sigma_{x}$ as initial state at finite temperature $\bar{n}=1.0$. Solid lines show results for the QO model, square points those for the DH model. The color coding identifies the different dissipation rates: $\kappa / g=1.0$ (red), 10.0 (blue), 20 (yellow), and 40 (black). For the DH model, all rates have been reduced by one half. The upper (lower) panel show the case of large, $(\Delta-1) / g=0.8($ small, $(\Delta-1) / g=0.1)$ detuning.

initial state, as defined in Eq. (10). In distinction to the zero-temperature case, the $\mathrm{DH}$ result is no longer equal to the QO result, but they become equal as $\kappa / g$ becomes large. The stabilization effect works as efficient as in the zero temperature case (Fig. 3). In Fig. 6, we show the differences between the $\mathrm{QO}$ and $\mathrm{DH}$ results from Fig. (5). Again, we find the expected convergence to the DH model for strong coupling.

\section{Dephasing model at finite temperature}

In this section, we replace the JC coupling between the qubit and the cavity mode by the dephasing coupling, Eq. (3), where the diagonal matrix elements of the qubit state remain constant. Without external heat bath, the coherence (non-diagonal element of the atom state) measures the fidelity amplitude for the Hamiltonian of the cavity mode, perturbed by the cavity-term of
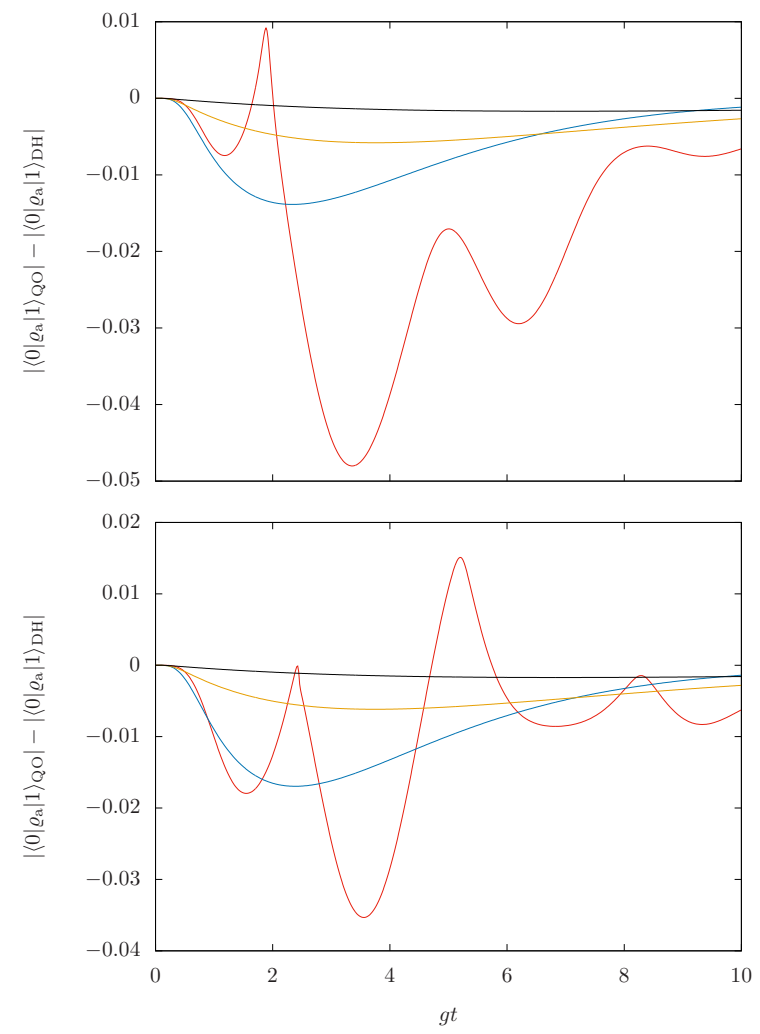

FIG. 6. (Color online) Differences between the curves of the Fig. (5), using the color coding from there.

the dephasing coupling [30, 31].

For random matrix models, the stabilization effect for the coherence has been demonstrated in Refs. [20, 21]. There, it was found that the stabilization effect is efficient for small dephasing couplings, only, i.e. $2 \pi g$ must be small as compared to the level spacing in the near environment (perturbative regime). In that case, the dissipation due to the heat bath suppresses the decoherence, just as in the cases shown here. For larger couplings $g$, the fidelity decays faster, eventually faster than the Heisenberg time of the near environment. Then, there is not enough time for the dynamics to "realize" that the system is finite (discrete spectrum), which avoids any effect when adding dissipation. This becomes understandable, if one interprets the increase of the coupling as a way to enlarge the near environment.

In Fig. 7, we show our results for the $\mathrm{QO}$ and the $\mathrm{DH}$ dissipation model. Results for the CL model have been analyzed also, but they are not shown as they agree very well with the QO model (in the regime $\kappa \leq 1$, where the CL model is applicable).

As explained above, the level spacing in the intermediate system determined by $w_{\mathrm{e}}$ is now important, providing an additional energy scale. If the stabilization effect would work similarly as in the random matrix cases, we would expect to find the effect for small values of $g$ and $\kappa / g \gg 1$. This is clearly not the case, as can be seen 

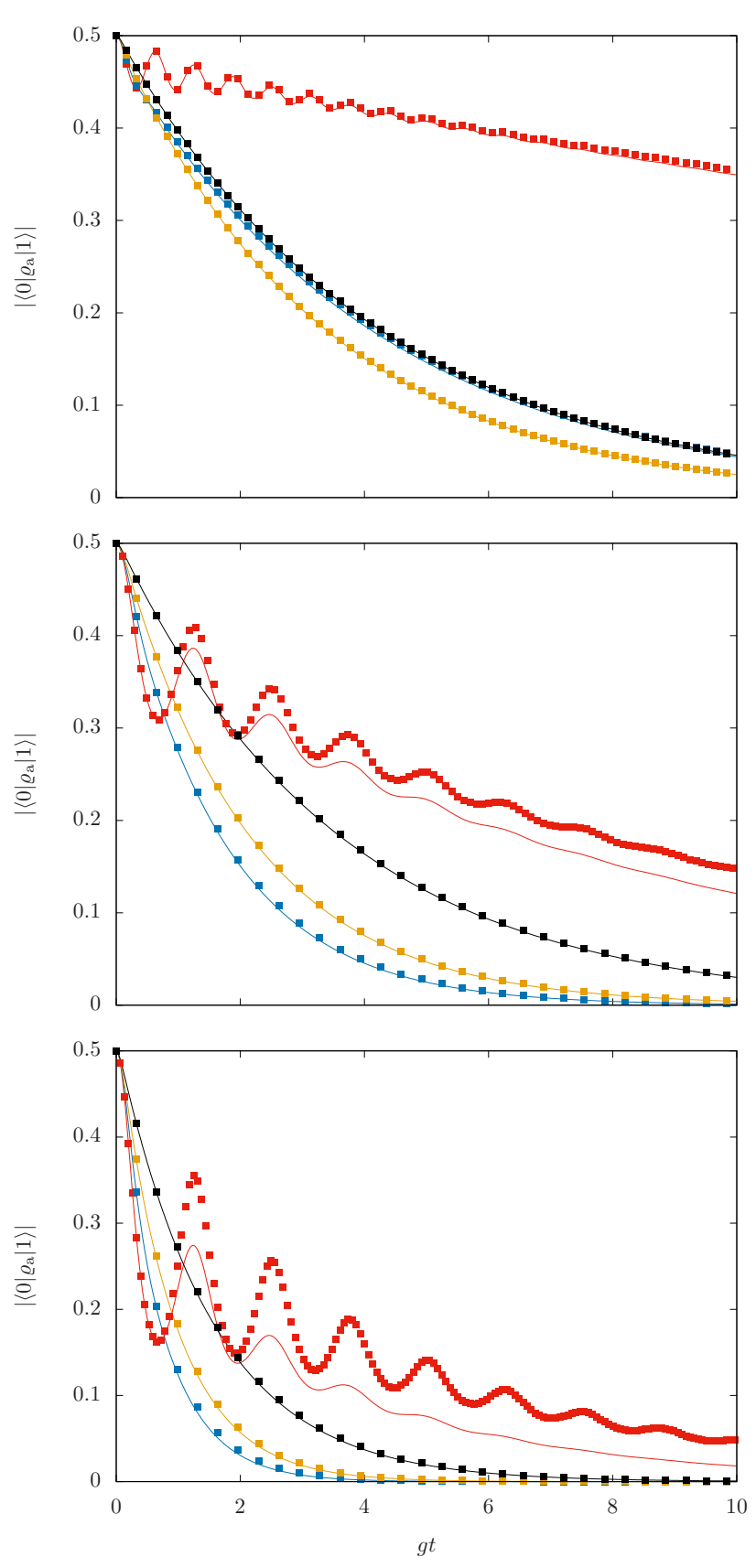

FIG. 7. (Color online) The coherence for an eigenstate of $\sigma_{x}$ as initial state for the dephasing coupling between qubit and near environment. Solid lines show results for the QO dissipation model, square points for the DH model. The color coding identifies the different dissipation rates: $\kappa / g=1.0$ (red), 10.0 (blue), 20 (yellow), and 40 (black). For the DH model, all rates have been reduced by one half. Upper panel: Temperature $\bar{n}=1.0$, dephasing coupling $g=0.1$. Middle panel: same temperature but larger coupling, $g=0.2$. Lower panel: larger coupling $g=0.2$ and larger temperature $\bar{n}=$ 3.0. in the upper panel, where $g=0.1$. Up to $\kappa / g>10$ no stabilization effect can be observed, and only between $\kappa / g=20$ (yellow lines) and $\kappa / g=40$ (black line) some stabilization effect may seem to set in.

What is even more surprising, for the larger values of $g$ (e.g. $g=0.2$ as in the middle panel), the stabilization effect seem to set in even earlier. There we find the turnover between $\kappa / g=10$ and $\kappa / g=20$. This is confirmed in the lower panel, where the value for $g$ is the same, but the temperature was increased to $\bar{n}=3.0$. In this case, the turnover is again observed between $\kappa / g=10$ and $\kappa / g=20$. Though note that the overall decoherence rate is increased due to the higher temperature.

\section{CONCLUSIONS}

We introduced the depolarizing heat bath $(\mathrm{DH})$ as a minimal dissipation model, which fulfills all the basic requirements of a heat bath. We use this model to study the relaxation of a central system, coupled via an intermediate system (the near environment) to that heat bath. At strong dissipation, i.e. if the dissipation rate is larger than the coupling between central system and near environment, the DH model seems to become universal in the following sense:

Consider a bipartite system, consisting of a central system and a near environment, coupled to an outer heat bath. Assume that its relaxation process is described by some quantum Markovian master equation, with dissipation terms which involve the near environment degree(s) of freedom, only. Assume further, that we study the relaxation of the central system, when the initial state is a product state with the near environment in thermal equilibrium with the heat bath. Then we conjecture that in the strong coupling case, the dynamics of the central system can be described equally well with the help of the $\mathrm{DH}$ dissipation model.

Previously, this has been shown for bipartite and tripartite random matrix models $[20,21]$ in the limit of infinite temperature. Here, we give numerical evidence for a bipartite model consisting of a two-level system and a harmonic oscillator mode, with Jaynes-Cummings or dephasing coupling, and using two different fundamental dissipation models (the quantum optical and the Caldeira-Leggett model) to describe the coupling between the oscillator mode and a heat bath.

A bipartite quantum system appears naturally, in the pseudo-mode theory developped in [5], and generalizations to the case of multiple modes [6,7], which lead to Markovian master equations on the expense of enlarging that part of the system which is described by Hamiltonian dynamics. We may thus divide the system into two parts: the "central system" which contains those degrees of freedom were are interested in, and the remaining (added) degrees of freedom, we call the "intermediate system". We found numerical evidence that such dissipa- 
tive bi-partite systems, may be described by a universal dissipation model (the depolarizing heat bath), when the coupling to the external heat bath (dissipation) is sufficiently strong.

In that limit the coupling to the heat bath may be considered as an ideal thermal contact and thus, a setup as described here, may be useful for the study of quantum thermal machines, as it may provide an alternative method for defining or determining thermodynamic quantities such as heat and work.

\section{ACKNOWLEDGMENTS}

We are greateful for very fruitful discussions with A. Eisfeld, P. C. López, J. M. Torres and T. H. Seligman. ABR and TG acknowledge the hospitality of the MaxPlanck-Institut for the Physics of complex systems, as well as the Centro Internacional de Ciencias, where many of these discussions took place.

\section{Appendix A: Thermal equilibrium states of the harmonic oscillator}

As far as the temperature in the diffusion term is concerned, note that for the harmonic oscillator state $w_{T}$ in thermal equilibrium, it holds

$$
\begin{aligned}
w_{T} & =\sum_{n=0}^{\infty} \varrho_{n n}|n\rangle\langle n|, \quad \varrho_{n n}=\frac{\mathrm{e}^{-b n}}{Z}, \quad \text { where } \\
b & =\frac{\hbar w_{\mathrm{e}}}{k_{\mathrm{B}} T} \quad \text { and } \quad Z=\sum_{n=0}^{\infty} \mathrm{e}^{-b n}=\frac{1}{1-\mathrm{e}^{-b}} .
\end{aligned}
$$

Therefore,

$$
\begin{aligned}
\bar{n} & =\frac{1}{Z} \sum_{n=0}^{\infty} n \mathrm{e}^{-b n}=\frac{-\partial_{b} Z}{Z}=-\partial_{b} \ln (Z)=\partial_{b} \ln \left(1-\mathrm{e}^{-b}\right) \\
& =\frac{\mathrm{e}^{-b}}{1-\mathrm{e}^{-b}}=\frac{\mathrm{e}^{-b / 2}}{\mathrm{e}^{b / 2}-\mathrm{e}^{-b / 2}}=\frac{\operatorname{coth}(b / 2)-1}{2} \\
& \Rightarrow \quad \operatorname{coth}\left(\frac{\hbar w}{2 k_{B} T}\right)=2 \bar{n}+1 .
\end{aligned}
$$

\section{Appendix B: Caldeira-Leggett model in original variables}

The original CL-model $[4,18]$, has the following master equation (we use the notation of Ref. [32])

$i \hbar \partial_{\tau} \varrho=\hbar w_{\mathrm{e}}\left[a^{\dagger} a, \varrho\right]+\gamma[\hat{X},\{\hat{P}, \varrho\}]-2 i \gamma \frac{m_{\mathrm{e}} k_{\mathrm{B}} T}{\hbar}[\hat{X},[\hat{X}, \varrho]]$,

where $\tau$ denotes the original time in physical units. Replacing the physical position and momentum operators by adimensional ones, i.e. $\hat{X}=\sqrt{\hbar /\left(m_{\mathrm{e}} w_{\mathrm{e}}\right)} \hat{x}$ and $\hat{P}=\sqrt{\hbar m_{\mathrm{e}} w_{\mathrm{e}}} \hat{p}$ we find

$$
i \partial_{\tau} \varrho=w_{\mathrm{e}}\left[a^{\dagger} a, \varrho\right]+\gamma[\hat{x},\{\hat{p}, \varrho\}]-i \gamma \frac{2 k_{\mathrm{B}} T}{\hbar w_{\mathrm{e}}}[\hat{x},[\hat{x}, \varrho]] .
$$

In order to deal gracefully with the low temperature regime, we follow the original work [18], and consider the ratio $2 k_{\mathrm{B}} T /\left(\hbar w_{\mathrm{e}}\right)$ as the high temperature limit of

$$
\operatorname{coth}\left(\frac{\hbar w}{2 k_{\mathrm{B}} T}\right)=2 \bar{n}+1
$$

where $\bar{n}$ is the average number of excited modes of the harmonic oscillator at thermal equilibrium. Finally, we switch to the adimensional time $t=w_{\mathrm{e}} \tau$ and thus obtain:

$$
\begin{aligned}
i \partial_{t} \varrho=w_{\mathrm{e}}\left[\hat{a}^{\dagger} \hat{a}, \varrho\right] & +\gamma[\hat{x},\{\hat{p}, \varrho\}] \\
& -i \gamma(2 \bar{n}+1)[\hat{x},[\hat{x}, \varrho]]
\end{aligned}
$$

where $\gamma$ is the classical damping rate, i.e. the system loses energy with that rate. Comparing to the master equation with the general form, Eq. (1) in Sec. II, to find

$$
\mathcal{D}_{\mathrm{CL}}[\varrho]=2 i[\hat{x},\{\hat{p}, \varrho\}]+2(2 \bar{n}+1)[\hat{x},[\hat{x}, \varrho]] .
$$

[1] K. Huang. Statistical Mechanics. (John Wiley \& Sons, New York, 1987).

[2] V. Gorini, A. Kossakowski, and E. C. G. Sudarshan, Completely positive dynamical semigroups of n-level systems, Journal of Mathematical Physics 17, 821 (1976).

[3] G. Lindblad, On the Generators of Quantum Dynamical Semigroups, Communications in Mathematical Physics 48119 (1976).
[4] H.-P. Breuer and F. Petruccione, The Theory of open quantum systems, Oxford University Press, Oxford, (2002).

[5] B. M. Garraway, Nonperturbative decay of an atomic system in a cavity, Physical Review A: Atomic, Molecular, and Optical Physics 55, 2290 (1997).

[6] B. J. Dalton, S. M. Barnett, and B. M. Garraway, Theory of pseudomodes in quantum optical processes, Physical 
Review A: Atomic, Molecular, and Optical Physics 64, 053813 (2001).

[7] J. Roden, W. T. Strunz, K. B. Whaley, and A. Eisfeld, Accounting for intra-molecular vibrational modes in open quantum system description of molecular systems, The Journal Chemical Physics 137, 204110 (2012).

[8] P. P. Hofer, M. Perarnau-Llobet, L. D. M. Miranda, G. Haack, R. Silva, J. B. Brask and N. Brunner, Markovian master equations for quantum thermal machines: local versus global approach, New Journal of Physics 19, 123037 (2017).

[9] T. Gorin, C. Pineda, H. Kohler, and T. H. Seligman, A random matrix theory of decoherence, New Journal of Physics 10, 115016 (2008).

[10] P. Skrzypczyk, N. Brunner, N. Linden, S. Popescu, The smallest refrigerators can reach maximal efficiency, Journal of Physics A: Mathematical and Theoretical 44, 492002 (2011).

[11] J. Bohr Brask,G. Haack, N. Brunner, and M. Huber, Autonomous quantum thermal machine for generating steady-state entanglement, New Journal of Physics, 17 113029 (2015).

[12] E. T. Jaynes and F. W. Cummings, Comparison of quantum and semiclassical radiation theories with application to the beam maser, Proceedings of the IEEE 51, 89 (1963).

[13] S. M. Barnett and P. L. Knight, Dissipation in a fundamental model of quantum optical resonance, Physical Review A: Atomic, Molecular, and Optical Physics 33, 2444 (1986).

[14] R. R. Puri, Mathematical Methods of Quantum Optics, (Springer, Berlin, 2001).

[15] J. M. Raimond, M. Brune, and S. Haroche, Colloquium: manipulating quantum entanglement with atoms and photons in a cavity, Reviews of Modern Physics 73, 565 (2001).

[16] A. D. Greentree, J. Koch, and J. Larson, Fifty years of Jaynes-Cummings physics, Journal of Physics B: Atomic, Molecular and Optical Physics 46, 220201 (2013).

[17] R. Betzholz, J. M. Torres, and M. Bienert, Quantum optical master equation for solid-state quantum emitters, Physical Review A: Atomic, Molecular, and Optical Physics 90, 063818 (2014).

[18] A. O. Caldeira and A. J. Leggett, Path integral approach to quantum brownian motion, Physica 121A, 587 (1983).

[19] B. L. Hu, J. P. Paz, and Y. Zhang, Quantum brownian motion in a general environment: exact master equa- tion with nonlocal dissipation and colored noise, Physical Review D: Covering Particles, Fields, Gravitation, and Cosmology 45, 2843 (1992).

[20] H. J. Moreno, T. Gorin, and T. H. Seligman, Improving coherence with nested environments, Physical Review A: Atomic, Molecular, and Optical Physics 92, 030104 (2015).

[21] T. Gorin, H. J. Moreno, and T. H. Seligman, A generalized fidelity amplitude for open systems, Philosophical Transactions of the Royal Society of London A:Mathematical, Physical and Engineering Sciences 374, (2016).

[22] J. I. Cirac, Interaction of a two-level atom with a cavity mode in the bad-cavity limit, Physical Review A: Atomic, Molecular, and Optical Physics 46, 4354 (1992).

[23] K. M. Fonseca-Romero, Decoherencia atómica en una zona de Ramsey fría, Momento 37, 1 (2008). issn electronico: 2500-8013. issn impreso: 0121-4470.

[24] J. M. Torres and T. H. Seligman, Protecting coherence by environmental decoherence: a solvable paradigmatic model, New Journal of Physics 19, 113016 (2017).

[25] C. González-Gutiérrez, E. Villaseñor, C. Pineda and T. H. Seligman, Stabilizing coherence with nested environments: a numerical study using kicked Ising models, Physica Scripta 91, 083001 (2016).

[26] A. Sandulescu and H. Scutaru, Open quantum systems and the damping of collective modes in deep inelastic collisions, Annals of Physics 173, 0003 (1987).

[27] L. Diosi, On High-Temperature Markovian Equation for Quantum Brownian Motion, Europhysics Letters 22, 1 (1993).

[28] F. M. Ramazanoglu, On High-Temperature Markovian Equation for Quantum Brownian Motion, Physical Review A: Atomic, Molecular, and Optical Physics 42, 265303 (2009).

[29] G. J. Milburn, Kicked quantized cavity mode: An opensystems-theory approach, Physical Review A: Atomic, Molecular, and Optical Physics 36, 744 (1987).

[30] S. A. Gardiner, J. I. Cirac, and P. Zoller, Quantum chaos in an ion trap: the delta-kicked harmonic oscillator, Physical Review Letters 79, 4790 (1997).

[31] T. Gorin, T. Prosen, T. H. Seligman, and W. T. Strunz, Connection between decoherence and fidelity decay in echo dynamics, Physical Review A: Atomic, Molecular, and Optical Physics 70, 042105 (2004).

[32] M. Á. Prado Reynoso, P. C. López Vázquez, and T. Gorin, Quantum kicked harmonic oscillator in contact with a heat bath, Physical Review A: Atomic, Molecular, and Optical Physics 95, 022118 (2017). 